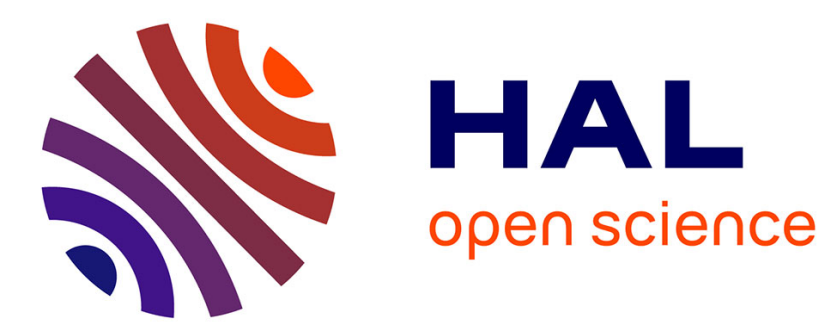

\title{
A fractional Burgers equation arising in nonlinear acoustics: theory and numerics.
}

Bruno Lombard, Denis Matignon, Yann Le Gorrec

\section{To cite this version:}

Bruno Lombard, Denis Matignon, Yann Le Gorrec. A fractional Burgers equation arising in nonlinear acoustics: theory and numerics.. 9ème Symposium IFAC sur les Systèmes de Commande Non-Linéaires, NOLCOS'13., Sep 2013, Toulouse, France. pp.1-6. hal-00910936

\section{HAL Id: hal-00910936 https://hal.science/hal-00910936}

Submitted on 28 Nov 2013

HAL is a multi-disciplinary open access archive for the deposit and dissemination of scientific research documents, whether they are published or not. The documents may come from teaching and research institutions in France or abroad, or from public or private research centers.
L'archive ouverte pluridisciplinaire HAL, est destinée au dépôt et à la diffusion de documents scientifiques de niveau recherche, publiés ou non, émanant des établissements d'enseignement et de recherche français ou étrangers, des laboratoires publics ou privés. 


\title{
A fractional Burgers equation arising in nonlinear acoustics: theory and numerics *
}

\author{
Bruno Lombard ${ }^{*}$ Denis Matignon ${ }^{* *}$ Yann Le Gorrec ${ }^{* * *}$ \\ * LMA, CNRS UPR 7051; 31 chemin Joseph Aiguier, 13402 Marseille, \\ France. lombard@lma.cnrs-mrs.fr. \\ ** University of Toulouse, ISAE; 10, av. Edouard Belin; BP 54032. \\ 31055 Toulouse Cedex 4, France. denis.matignon@isae.fr. \\ *** FEMTO-ST institute, ENSMM 24, rue Alain Savary, 25000 \\ Besançon, France. Yann.Le.Gorrec@ens2m.fr.
}

\begin{abstract}
The study of a fractional Burgers equation arising in nonlinear acoustics is presented. The motivation comes from an elementary model of shock waves in brass wind instruments, that proves useful in musical acoustics. Such a model results from the coupling of a conservative nonlinear system with a dissipative term; here the dissipation is represented by a fractional derivative in time, for which equivalent diffusive representations can be efficiently used: in a first part, strong solutions, weak solutions and energy balances are examined. In a second part, ad hoc numerical schemes are derived, in order to capture all the physical phenomena at stake in the original model, and to get rid, as far as possible, of the spurious numerical effects which are highly undesirable: to this end, conservative schemes for hyperbolic conservation laws, diffusive realizations for the fractional derivatives and integrals, and splitting of the two are being used.
\end{abstract}

Keywords: fractional derivatives, Burgers equation, diffusive representation, numerical methods.

\section{INTRODUCTION}

The motivation of this work comes from musical acoustics, the underlying idea being to model wind instruments from the brass family, well-known for their brassy effects, which is a typically nonlinear phenomenon. Moreover, shock waves can be developped after some critical time, depending on the amplitude of the input or of the initial condition.

Such purely nonlinear effect can be obtained by means of solution to nonlinear PDEs, such as the inviscid Burgers equation: this equation has been widely studied, both from theoretical aspect (hyperbolic equation, conservation law) and numerical aspect (conservative schemes, such as Godunov).

Now, a specific feature of wind instruments is the fact that viscous and thermal effects do play a role at the lateral walls of the duct, and contrarily to the open space case, the bounded space imposes a very specific behaviour, see Bruneau et al. [1989] and Polak [1991]: evolution as the square root of the frequency, with much bigger physical coefficients, which translate into non negligible effects; to put it shortly, the presence of the boundary layer can almost be listened to! From a modelling point of view, the classical wave PDE is modified with a fractional derivative in time, this behaves as a damping term with long-memory decay, see e.g. Lokshin [1978], Lokshin and Rok [1978].

\footnotetext{
* Part of this work has been done within the context of the French National Research Agency sponsored project HAMECMOPSYS. Further information is available at http://www . hamecmopsys.ens $2 \mathrm{~m} . \mathrm{fr} /$.
}

The one-way Burgers-Lokshin (BL) equation is the simplest model, that combines both these features (nonlinearity and fractional derivative), it has the following form:

$$
\partial_{t} u+c \partial_{x} u+\varepsilon \partial_{t}^{\alpha} u+b \partial_{x}\left(u^{2} / 2\right)=0 \text { for } t>0,
$$

with compactly supported initial datum $u(t=0, x)=$ $u^{0}(x)$ at $t=0$. The coefficients are $c>0$ the sound speed, and $\varepsilon>0$ which takes into account the specific length of both viscous and thermal effects and the radius of the duct; moreover the fractional order $\alpha \in(0,1)$ is $\alpha=\frac{1}{2} ; b \geq 0$, or Burgers coefficient, quantifies the nonlinear effects.

Accounting for the existing literature on this sharp subject is not so easy, but we should mention first Makarov and Ochmann [1997] for a general overview, then Menguy and Gilbert [2000] for a case very close to ours, and finally Hélie and Hasler [2004], Hélie and Smet [2008] for explicit solutions of this latter case, using Volterra series. The paper is organized as follows: in $\S 2$, strong solutions and weak solutions are defined, and a useful energy balance is proved. Then in $\S 3$, numerical methods are presented, that allow for efficient simulation of the oneway Burgers-Lokshin equation, taking advantage of the two different underlying structures: nonlinear hyperbolic equation, together with a linear diffusive system; in $\S 4$, the results of numerical experiments are presented and carefully explained. Finally in $\S 5$ a conclusion is drawn on this work, and many perspectives and open questions are proposed: one concerns the proof of existence of weak solutions to the original model, e.g. in the case of discontinuous inital data, or after a shock has appeared, i.e. when no more strong solutions can be expected. In order to be self-contained, Appendix A recalls definitions 
of fractional integrals and derivatives, together with their diffusive representations.

\section{THEORETICAL RESULTS}

\subsection{Strong solutions}

Let us first recall the following theorem, proved in Haddar and Matignon [2004]:

Theorem 1. Let $\mathcal{H}$ be a Hilbert space, and $A: D(A) \subset$ $\mathcal{H} \rightarrow \mathcal{H}$ be a maximal monotone operator, and $F$ some non-linear function $F: \mathcal{H} \rightarrow \mathcal{H}$, such that the semi-linear evolution problem

$$
\partial_{t} X+A X=F(X), \quad t>0,
$$

with $X(0)=X_{0} \in D(A)$ is well-posed, for $t \in\left[0, T_{\text {max }}\right)$, meaning the existence and uniqueness of a strong solution $X \in C^{1}\left(\left[0, T_{\max }\right) ; \mathcal{H}\right) \cap C^{0}\left(\left[0, T_{\max }\right) ; D(A)\right)$.

Then, for any two positive pseudo-differential operators (PDOs) of diffusive type, such as the fractional integral $I^{\beta}$ in $\S A .1$, or as the fractional derivative $D^{\alpha}$ in $\S A .2$, the pseudo-differential non-linear problem:

$$
\partial_{t} X+I^{\beta} X+D^{\alpha} X+A X=F(X), \quad t>0,
$$

with $X(0)=X_{0} \in D(A)$, has also a unique strong solution $X \in C^{1}\left(\left[0, T^{\prime}{ }_{\max }\right) ; \mathcal{H}\right) \cap C^{0}\left(\left[0, T^{\prime}{ }_{\max }\right) ; D(A)\right)$ with $T_{\max }^{\prime} \geq T_{\max }$.

Remark 1. For instance, the well-posedness of problem (2) is garanteed in the case when $F$ is locally Lipschitz on $\mathcal{H}$, see [Pazy, 1983, Theorem 1.4 in ch. 6].

Remark 2. Even though $u \mapsto F(u)=u^{2} / 2$ is locally Lipschitz, care must be taken not to make use the above theorem too rapidly: unfortunately, equation (1) does not fit in this framework, since the problem is nonlinear both in $u$ and $\partial_{x} u$.

Some adequate formulation is still to be found in order to give existence and uniqueness results on strong solutions to equation (1).

\subsection{Weak solutions}

Moreover, since it is known that, even with $C^{\infty}$ initial datum $u^{0}$, a shock (i.e. a discontinuity) may occur for $t>0$ (typically at $t=T_{\max }$ or $t=T_{\max }^{\prime}$ ), it is necessary to define generalized or weak solutions to (1).

Definition 1. A weak solution to (1) is a function $u \in$ $L_{\text {loc }}^{1}\left(\mathbb{R}^{+} \times \mathbb{R}\right)$, such that $u^{2} \in L_{\text {loc }}^{1}\left(\mathbb{R}^{+} \times \mathbb{R}\right)$, and $\forall \phi \in$ $C_{c}^{1}((-1,+\infty) \times \mathbb{R})$, the following identity holds:

$$
-\int_{\mathbb{R}} u^{0}(x) \phi(0, x) \mathrm{d} x=
$$

$\int_{0}^{\infty} \int_{\mathbb{R}}\left(u \partial_{t} \phi+c u \partial_{x} \phi+b\left(u^{2} / 2\right) \partial_{x} \phi-\varepsilon u . \check{h}_{1-\alpha} \star \partial_{t} \phi\right) \mathrm{d} x \mathrm{~d} t$.

where $\breve{h}(t)=h(-t)$, the convolution on $\partial_{t} \phi$ becoming anticausal in the weak formulation.

Proposition 1. Any strong solution is necessarily a weak solution (the proof goes classically by integration by parts), whereas the converse is true only under regularity assumptions on the solution.

Remark 3. Existence of weak solution is still an open question. Also finding compatibility conditions, such as Rankine-Hugoniot conditions, is an open question.

\subsection{Energy balance}

Making use of appendix A.2, an energy density can be defined as follows: $e_{\tilde{\varphi}}(t, x):=\frac{1}{2} \int_{0}^{\infty} \xi \mu_{1_{\alpha}}(\xi) \widetilde{\varphi}(\xi, t, x)^{2} \mathrm{~d} \xi$. Then, with candidate energy function for equation (1)

$$
\mathcal{E}_{A B}(t):=\int_{A}^{B} \frac{1}{2} u(t, x)^{2}+\varepsilon e_{\widetilde{\varphi}}(t, x) \mathrm{d} x,
$$

the following energy balance can be proved:

$$
\begin{aligned}
\frac{d}{d t} \mathcal{E}_{A B}(t)= & -\int_{A}^{B} \varepsilon \int_{0}^{\infty} \mu_{1_{\alpha}}(\xi)\left(\partial_{t} \widetilde{\varphi}(\xi, t, x)\right)^{2} \mathrm{~d} \xi \mathrm{d} x \\
& +\left[c \frac{u^{2}(t, x)}{2}+b \frac{u^{3}(t, x)}{6}\right]_{A}^{B} .
\end{aligned}
$$

Proposition 2. When $u^{0}$ is compactly supported and smooth enough, thanks to finite propagation speed, it can be inferred that equation (1) is dissipative on $(A, B)=\mathbb{R}$.

\section{NUMERICAL METHOD}

Even though the theory for this equation seems not to be sufficiently mature so far, a numerical strategy has been adopted to solve (1), as follows:

(1) use a conservative numerical scheme for hyperbolic conservation laws, like Godunov, Total Variation Diminishing (TVD) or Weighted Essentially NonOscillatory (WENO),

(2) use Diffusive Representation (DR), that help transform fractional derivatives and integral into a system of linear ODEs,

(3) use splitting, before coupling with $\frac{d u}{d t}$; this system is solved exactly in order to avoid numerical instabilities.

\subsection{A semi-discretized version for simulation}

For $0<\alpha<1$, using appendix A for classical diffusive representations (see e.g. Hélie and Matignon [2006]), or following Diethelm [2008], upon setting $\xi=\theta^{2}$, another exact diffusive representation of $\partial_{t}^{\alpha} u=I^{1-\alpha}\left(\partial_{t} u\right)$ can be given by:

$$
\begin{aligned}
& \partial_{t}^{\alpha} u(t)=\int_{0}^{+\infty} \phi(\theta, t) \mathrm{d} \theta \\
& \frac{d}{d t} \phi(\theta, t)=-\theta^{2} \phi+\gamma_{\alpha} \theta^{2 \alpha-1} \frac{\partial u}{\partial t} . \quad \phi(\theta, 0)=0,
\end{aligned}
$$

where we have set $\gamma_{\alpha}:=\frac{2 \sin \pi \alpha}{\pi}$. Then, the continuum (4) is discretized into

$$
\left\{\begin{array}{l}
d_{t}^{\alpha} u \approx \sum_{\ell=1}^{N} \mu_{\ell} \phi\left(\theta_{\ell}, t\right) \equiv \sum_{\ell=1}^{N} \mu_{\ell} \phi_{\ell}, \\
\frac{d \phi_{j}}{d t}=-\theta_{j}^{2} \phi_{j}+\gamma_{\alpha} \theta_{j}^{2 \alpha-1} \frac{\partial u}{\partial t}, \quad j=1, \cdots N, \\
\phi_{j}(0)=0 .
\end{array}\right.
$$

The number $N$ of relaxation mechanisms plays a role both on the accuracy of the approximation and also on the computing cost. The determination of weights $\mu_{\ell}$ and quadrature nodes $\theta_{\ell}$ is discussed further. Combining (1) 
and (5) yields the first-order system without fractional derivatives

$$
\left\{\begin{array}{l}
\frac{\partial u}{\partial t}+c \frac{\partial u}{\partial x}+b \frac{\partial}{\partial x}\left(\frac{u^{2}}{2}\right)=-\varepsilon \sum_{\ell=1}^{L} \mu_{\ell} \phi_{\ell}, \\
\frac{\partial \phi_{j}}{\partial t}+c_{\alpha} \theta_{j}^{2 \alpha-1}\left(c \frac{\partial u}{\partial x}+b \frac{\partial}{\partial x}\left(\frac{u^{2}}{2}\right)\right) \\
=-\theta_{j}^{2} \phi_{j}-c_{\alpha} \theta_{j}^{2 \alpha-1} \varepsilon \sum_{\ell=1}^{L} \mu_{\ell} \phi_{\ell} .
\end{array}\right.
$$

Taking the vector of $(N+1)$ unknowns

$$
\mathbf{U}=\left(u \phi_{1} \ldots \phi_{N}\right)^{T},
$$

the system (6) can be put in the form

$$
\frac{\partial}{\partial t} \mathbf{U}+\frac{\partial}{\partial x} \mathcal{F}(\mathbf{U})=\mathbf{S} \mathbf{U}
$$

where $\mathcal{F}=\left(\mathcal{F}_{1} \mathcal{F}_{2} \ldots \mathcal{F}_{N+1}\right)^{T}$ is the nonlinear flux function

$$
\mathcal{F}_{1}=c u+b \frac{u^{2}}{2}, \quad \mathcal{F}_{j}=\gamma_{\alpha} \theta_{j}^{2 \alpha-1} \mathcal{F}_{1}, \quad j=2 \cdots N+1,
$$

and $\mathbf{S}$ is the $(N+1) \times(N+1)$ diffusive matrix

$$
\mathbf{S}=-\left(\begin{array}{cccc}
0 & \varepsilon \mu_{1} & \cdots & \varepsilon \mu_{N} \\
0 & \theta_{1}^{2}+\Omega \mu_{1} & \cdots & \Omega \mu_{N} \\
\vdots & \vdots & \vdots & \vdots \\
0 & \Omega \mu_{1} & \cdots & \theta_{N}^{2}+\Omega \mu_{N}
\end{array}\right)
$$

with $\Omega=\gamma_{\alpha} \varepsilon$. The eigenvalues of the Jacobian matrix $\mathbf{J}=\mathcal{F}^{\prime}$ in (9) are real: $c+b u$, and 0 with multiplicity $2 N$. Remark 4. These eigenvalues do not depend on the coefficients of the diffusive representation.

\subsection{Numerical Modelling}

Numerical Scheme In order to integrate the system (8), one introduces a uniform mesh size $\Delta x$ and a variable time step $\Delta t$. The approximation of the exact solution $\mathbf{U}\left(x_{j}=j \Delta x, t^{n}=t^{n-1}+\Delta t\right)$ is denoted by $\mathbf{U}_{j}^{n}$. Unsplit integration of (8) is not optimal, because the time step stability condition typically implies

$$
\Delta t \leq \min \left(\frac{\Delta x}{c_{\max }}, \frac{2}{\varrho(\mathbf{S})}\right)
$$

where $c_{\max }:=c+b \max _{j}\left(u_{j}^{n}\right)$ is the maximum numerical velocity at time $t^{n}$, and $\mathbf{S}$ is the spectral radius of $\mathbf{S}$ which grows in an unbounded way with $N$, thus penalizing the standard CFL condition. Moreover, it requires to build an adequate scheme for the coupled system.

A more efficient strategy is adopted here, which consists in splitting the original system (8) into a propagative part (12a) and a diffusive part (12b)

$$
\left\{\begin{array}{l}
\frac{\partial}{\partial t} \mathbf{U}+\frac{\partial}{\partial x} \mathcal{F}(\mathbf{U})=\mathbf{0} \\
\frac{\partial}{\partial t} \mathbf{U}=\mathbf{S} \mathbf{U}
\end{array}\right.
$$

The discrete operators associated with steps (12a) and (12b) are denoted by $\mathbf{H}_{a}$ and $\mathbf{H}_{b}$, respectively. The secondorder Strang splitting (see e.g. LeVeque [2002], Holden et al. [2011]) is then used between $t^{n}$ ant $t^{n+1}$, leading to the time-marching scheme:

$$
\begin{aligned}
& \text { - } \mathbf{U}_{j}^{(1)}=\mathbf{H}_{b}\left(\frac{\Delta t}{2}\right) \mathbf{U}_{j}^{n}, \\
& \text { - } \mathbf{U}_{j}^{(2)}=\mathbf{H}_{a}(\Delta t) \mathbf{U}_{j}^{(1)}, \\
& \text { - } \mathbf{U}_{j}^{n+1}=\mathbf{H}_{b}\left(\frac{\Delta t}{2}\right) \mathbf{U}_{j}^{(2)} .
\end{aligned}
$$

The propagative part (12a) is solved by any standard scheme for nonlinear hyperbolic PDE:

$$
\begin{aligned}
& u_{j}^{n+1}=u_{j}^{n}-\frac{\Delta t}{\Delta x}\left(\mathcal{F}_{j+1 / 2}^{1}-\mathcal{F}_{j-1 / 2}^{1}\right), \\
& \phi_{j, \ell}^{n+1}=\phi_{j, \ell}^{n}-\gamma_{\alpha} \theta_{\ell}^{2 \alpha-1} \frac{\Delta t}{\Delta x}\left(\mathcal{F}_{j+1 / 2}^{1}-\mathcal{F}_{j-1 / 2}^{1}\right),
\end{aligned}
$$

where $\mathcal{F}_{j \pm 1 / 2}^{1}$ is the numerical flux function of the advection-Burgers part in (9). In practice, a second-order TVD scheme with MC-limiter is used in our numerical experiments, see LeVeque [2002]. Stability analysis of (14) yields the optimal CFL condition

$$
\Upsilon=\frac{c_{\max } \Delta t}{\Delta x} \leq 1
$$

Since the physical parameters do not vary with time, the diffusive part $(12 \mathrm{~b})$ can be solved exactly. This gives

$$
\mathbf{H}_{b}\left(\frac{\Delta t}{2}\right) \mathbf{U}_{j}=e^{\mathbf{S} \frac{\Delta t}{2}} \mathbf{U}_{j}
$$

It is computed numerically using a $(6,6)$ Padé approximation in the "scaling and squaring method", see Moler and Van Loan [2003]. If the physical parameters are constant in space, the computation is done only once at each time step, leading to a negligible computational cost. This part of the splitting is unconditionally stable, so that the global stability requirement is (15) and is not penalized by the diffusive part. In other words, the time step only depends on the advection and Burgers coefficients in (1). In particular, $\Delta t$ does not depend on the coefficients of the diffusive representation.

Coefficients of the Diffusive Representation It remains to determine the $2 N$ coefficients of the diffusive representation $\mu_{\ell}$ and $\theta_{\ell}$ in (10). This issue is crucial both for the accuracy of the modeling and for the computational efficiency of the method, see Hélie and Matignon [2006], and Deü and Matignon [2010]. Two strategies exist for this purpose.

The first one relies on Gaussian quadratures of improper integrals (4). A Laguerre quadrature formula can be used for this purpose Flannery et al. [1992]. This technique is frequently used in the literature, see e.g. Diethelm [2008], Birk and Song [2010], ensuring $\mu_{\ell}>0$ and $\theta_{\ell}>0$. We have tried this approach, but the convergence rate is very poor. As can be shown in the limit-case of the linear regime $(b=0)$, a large number of memory variables is required to get acceptable accuracy, which greatly increases the computational cost, see Appendix B.

When a characteristic length of the waves exists, a more efficient strategy can be proposed based on the Fourier relation (B.2). The original problem (1) and the first-order system (8) differ only in their symbol $\chi(\omega)$ : (B.3) in the first case, (B.7) in the second one. Adjusting them provides a mean to estimate $\mu_{\ell}$ and $\theta_{\ell}$. This technique is physically 

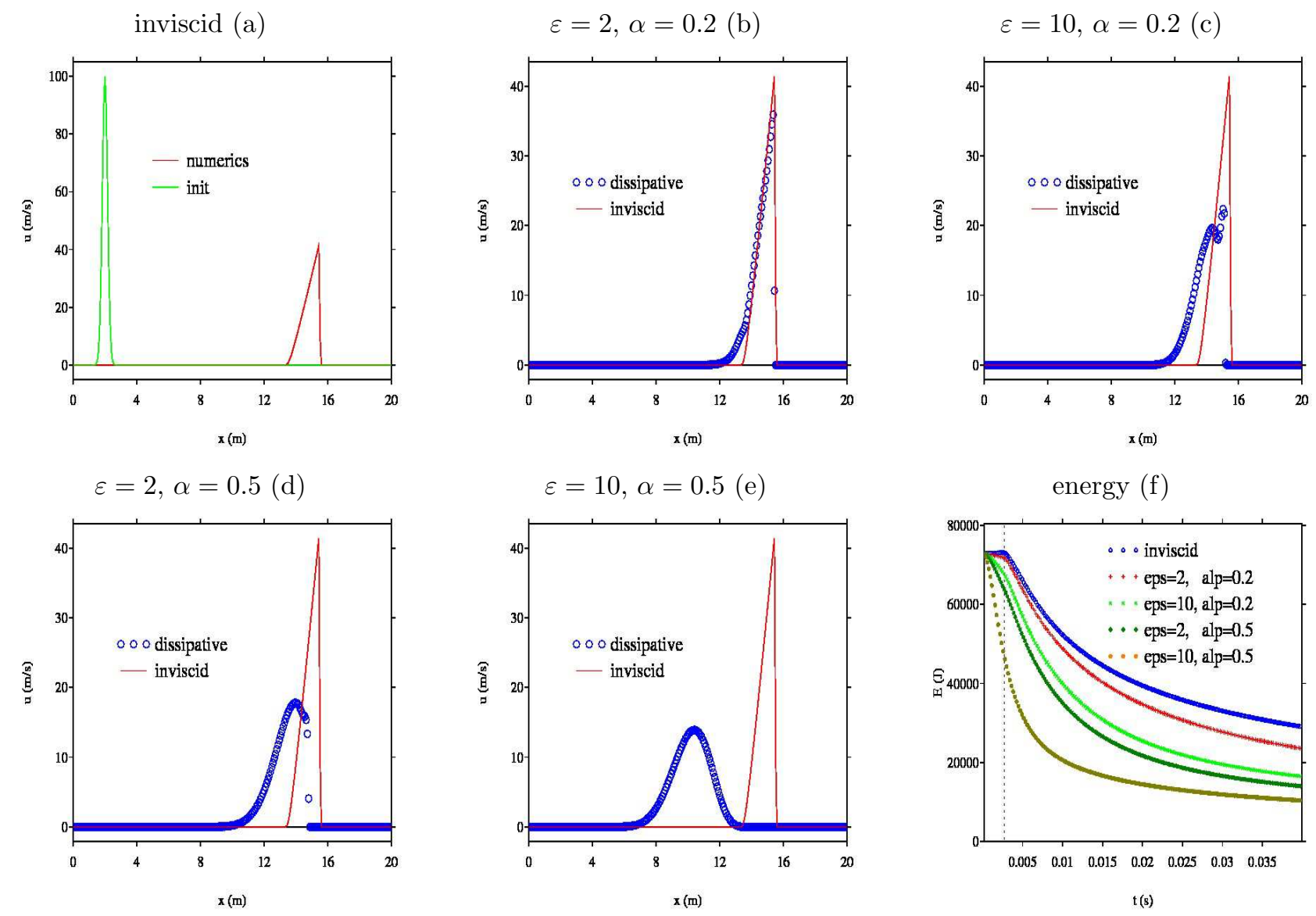

Fig. 1. Gaussian pulse. Snapshots of $u$ in the inviscid case and for various dissipative parameters $\varepsilon$ and $\alpha$ (a-e). The subfigure (f) shows the time evolution of the energy; the vertical dotted line denotes the time of break $t^{*}(18)$.

meaningful, and has proven its efficiency in a previous work about poroelastic waves, see Blanc et al. [2013].

\section{NUMERICAL EXPERIMENTS}

A $20 \mathrm{~m}$ long domain is discretized on 1000 grid nodes. The celerity of advection is $c=300 \mathrm{~m} / \mathrm{s}$, and the nonlinear coefficient is $b=1$ in (1). The CFL number is $\Upsilon=0.95$. One takes $N=6$ memory variables only in (7). The computations are initialized by a Gaussian pulse

$$
u_{0}(x)=u_{m} \exp \left(-\left(\frac{x-x_{0}}{\sigma}\right)^{2}\right),
$$

with amplitude of velocity $u_{m}=100 \mathrm{~m} / \mathrm{s}$, spatial shift $x_{0}=1 \mathrm{~m}$, wavelength $\lambda=1$, and standard deviation $\sigma=\frac{\lambda}{2 \sqrt{\ln 100}}$. In the inviscid case, the smooth Gaussian pulse develops a shock in finite time, yielding a decay of energy. The initial data give the time break

$$
t^{*}=\sqrt{\frac{e}{2}} \frac{\sigma}{b u_{m}}=0.021 \mathrm{~s} .
$$

Simulations are performed up to $t=0.04 \mathrm{~s}>t^{*}$, which corresponds to 769 steps in the inviscid case.

Figure 1 displays the simulations with the Gaussian pulse. In the inviscid case $\varepsilon=0$ (a), one shows the initial and the final solutions. Classically, the right part of the pulse has developped a shock. Dissipative models are examined in subfigures (b-e), for various values of $\varepsilon$ and $\alpha$ in (1); the numerical value of the inviscid case is shown in red line for comparison. As $\varepsilon$ and $\alpha$ increase, the amplitude of waves decreases, and the wave slows down. The shock is also smeared. For $\varepsilon=10$ and $\alpha=0.5$, the shock totally disappears. In subfigure (f), one shows the time evolution of the energy $E^{n}:=\sum_{j}\left(u_{j}^{n}\right)^{2}$, and the time break (18) is denoted by a vertical dotted line. In the inviscid case, the energy $E^{n}$ is constant up to $t^{*}$, and then it decreases. On the contrary, dissipation occurs at all times when $\varepsilon \neq 0$.

Another numerical experiment, made with a door pulse as initial condition, would show the following: in the inviscid case, the door pulse splits into two parts: a rarefaction fan and a right-going shock, which decreases the energy. These two waves interact at a specific time. Observations similar to the Gaussian case could be done: decrease of amplitude and slowdown of waves when $\varepsilon$ and $\alpha$ increase, smearing of shock.

\section{CONCLUSION AND PERSPECTIVES}

In this work, the following questions have been addressed: a fractional nonlinear PDE arising in musical acoustics has been studied, weak solutions have been properly defined, an energy balance has been proved. A numerical method 
to solve advection-Burgers equation with time fractional derivative has been proposed. Three parts are involved: a classical TVD scheme for nonlinear hyperbolic equations, a diffusive representation of the fractional derivatives to avoid storing past values of the solution, and a splitting to couple the previous tools in an efficient manner, see e.g. Lombard and Mercier [2013].

So far, many perspectives are left open:

- find an appropriate framework for existence and uniqueness of strong solutions.

- prove existence of weak solutions to the original model (in the sense of definition 1); it should be useful in at least two situations: in the case of discontinuous inital data, or after a shock has appeared, i.e. when no more strong solutions can be expected.

- recast this coupled model in the setting of infinitedimensional port-Hamiltonian systems (pHs) including dissipation. Based on the energy balance derived in $\S 2.3$, and following e.g. Maschke and van der Schaft [2011], one can choose as Hamiltonian functional $\mathcal{H}_{0}(u):=\int_{0}^{1}\left(c \frac{u^{2}}{2}+b \frac{u^{3}}{6}\right) \mathrm{d} z$ for the Burgers part; and using e.g. Le Gorrec and Matignon [2012], one can choose as Hamiltonian functional $\mathcal{H}_{\widetilde{\Phi}}:=$ $\frac{1}{2} \int_{0}^{\infty} \mu_{1-\alpha}(\xi) \xi \widetilde{\varphi}(\xi)^{2} \mathrm{~d} \xi$ for the diffusive part. So far, the port-Hamiltonian formulation of the infinitedimensional coupled system does not prove straightforward.

- ensure positivity of weights $\mu_{\ell}$ in the linear optimization procedure, since it is crucial for well-possedness of the diffusive model (6). To ensure $\mu_{\ell}>0$, we will implement optimization with constraint based on Shor's algorithm, see Rekik and Brenner [2011].

- perform a rigorous analysis of the shock zones, based e.g. on a matched asymptotic analysis as in Sugimoto [1991], to predict the width of the boundary layer. Indeed, depending on the parameters of the fractional terms, shocks are smeared or may even disappear, as shown by the numerical simulations

\section{ACKNOWLEDGEMENTS}

The authors would like to thank the three anonymous reviewers, who carefully read the original submission, made useful comments, and greatly helped enhance the final version of the paper; they are gratefully acknowledged.

\section{REFERENCES}

C. Birk AND C. Song, An improved non-classical method for the solution of fractional differential equations, Comput. Mech., 46:721-734, 2010.

E. Blanc, G. Chiavassa, and B. Lombard, Biot-JKD model: simulation of $1 \mathrm{D}$ transient poroelastic waves with fractional derivatives, J. Comput. Phys., 237:1-20, 2013.

M. Bruneau, Ph. Herzog, J. Kergomard, and J.D. POLACK, General formulation of the dispersion equation in bounded visco-thermal fluid, and application to some simple geometries, Wave Motion, 11:441-451, 1989.

J.-F. DeÜ AND D. MAtignon, Simulation of fractionally damped mechanical systems by means of a Newmarkdiffusive scheme, Computers and Mathematics with Applications, 59:1745-1753, 2010.
K. Diethelm, An investigation of some nonclassical methods for the numerical approximation of Caputotype fractional derivatives, Numer. Algor., 47:361-390, 2008.

B. P. Flannery, W. H. Press, S. A. Teukolsky, W. T. Vetterling, Numerical Recipes in $C$ : the Art of Scientific Computing, Second Edition, Cambridge University Press (1992).

E. Forest, R. D. Ruth, Fourth-order symplectic integration, Physica D, 43:105-117, 1990.

H. HADDAR AND D. MAtignon, Well-posedness of nonlinear conservative systems when coupled with diffusive systems, in IFAC symposium on Nonlinear Control Systems (NOLCOS), vol. 1, Stuttgart, Germany, 2004.

T. HÉlie AND M. HAsler, Volterra series for solving weakly nonlinear partial differential equations: Application to the Burgers equation with visco-thermal losses. Int. J. of Control, 77:1071-1082, 2004.

T. HÉLie And D. MATignon, Representations with poles and cuts for the time-domain simulation of fractional systems and irrational transfer functions. Signal Processing, 86:2516-2528, 2006.

T. HÉLIE AND V. SMET, Simulation of the weakly nonlinear propagation in a straight pipe: application to a realtime brassy audio effect. In 16th IEEE Mediterranean conf. on Control and Automation (MED'08), June 2527, 2008, Ajaccio, France. p. 1580 - 1585, (invited session).

H. Holden, K. H. Karlsen, N. H. Risebro, and T. TAO, Operator splitting for the KDV equation, Math. Comput., 80:821-846, 2011.

Y. Le Gorrec, And D. Matignon, Diffusive systems coupled to an oscillator: a Hamiltonian formulation. In IFAC conf. on Lagrangian and Hamiltonian Methods and Nonlinear Control (LHMNLC'12), August 29-31, 2012, Bertinoro, Italy. (invited session).

R. J. LeVEque, Finite Volume Methods for Hyperbolic Problems, Cambridge University Press (2002).

A. A LoKSHIN, Wave equation with singular retarded time. Dokl. Akad. Nauk SSSR, 240:43-46, 1978. (in Russian).

A. A. LoKshin And V. E. Rok. Fundamental solutions of the wave equation with retarded time. Dokl. Akad. Nauk SSSR, 239:1305-1308, 1978. (in Russian).

B. Lombard, J. F. Mercier, Numerical modeling of nonlinear acoustic waves in a tube with an array of Helmholtz resonators, submitted to J. Comput. Phys., 2013.

S. MAKArov And M. OChmann. Nonlinear and thermoviscous phenomena in acoustics. Part II. Acta Acustica 83:199-222,1997.

B. MaschKe AND A.J. VAn DER SCHAFt. On alternative Poisson brackets for fluid mechanical systems and their extension to Stokes-Dirac structures. In: 7th Workshop on control of Distributed Parameter Systems, Wuppertal, 2011. (Invited Lecture)

L. Menguy And J. Gilbert. Weakly nonlinear gas oscillations in air-filled tubes; solutions and experiments. Acta Acustica, 86:798-810, 2000.

C. B. Moler, C. F. Van Loan, Nineteen dubious ways to compute the exponential of a matrix, twenty-five years later, SIAM Review, 45:3-49,2003.

A. PAZY, Semigroups of linear operators and applications 
to partial differential equations. Vol. 44 of Applied Mathematical Sciences. Springer Verlag, 1983.

J.-D. PoLACK, Time-domain solution of Kirchhoff's equation for sound propagation in viscothermal gases: a diffusion process. J. Acoustique, 4:47-67, 1991.

A. Rekik, R. Brenner, Optimization of the collocation inversion method for the linear viscoelastic homogenization, Mech. Res. Comm. 38:305-308, 2011.

N. Sugimoto, Burgers equation with a fractional derivative; hereditary effects on nonlinear acoustic waves, $J$. Fluid. Mech., 225:631-653, 1991.

\section{Appendix A. FRACTIONAL DERIVATIVES AND DIFFUSIVE REPRESENTATIONS}

\section{A.1 Fractional integral}

Let $u \in L^{2}(0, T)$, for any $\beta \in(0,1)$, define the causal convolution kernel $h_{\beta}(t):=\frac{1}{\Gamma(\beta)} t^{\beta-1}$ for $t>0$, then the fractional integral of order $\beta \in(0,1)$ of $u$ is defined by $I^{\beta} u:=h_{\beta} \star u$.

Since $h_{\beta}(t)=\int_{0}^{\infty} \mu_{\beta}(\xi) e^{-\xi t} \mathrm{~d} \xi$, with specific weight $\mu_{\beta}(\xi)=\frac{\sin (\beta \pi)}{\pi} \xi^{-\beta}$, the fractional integral can be reformulated by the following input-output representation :

$$
y(t)=\int_{0}^{\infty} \mu_{\beta}(\xi)\left[e_{\xi} \star u\right](t) \mathrm{d} \xi
$$

with $e_{\xi}(t):=e^{-\xi t}$, and $\left[e_{\xi} \star u\right](t)=\int_{0}^{t} e^{-\xi(t-\tau)} u(\tau) \mathrm{d} \tau$. The following infinite-dimensional dynamical system can be seen as a state-space realization of the fractional intergral of order $\beta$ :

$$
\begin{aligned}
\partial_{t} \varphi(\xi, t) & =-\xi \varphi(\xi, t)+u(t), \varphi(\xi, 0)=0 \\
y(t) & =\int_{0}^{\infty} \mu_{\beta}(\xi) \varphi(\xi, t) \mathrm{d} \xi
\end{aligned}
$$

\section{A.2 Fractional derivative}

For short, fractional derivative of order $\alpha \in(0,1)$ of $u$ in a generalized sense is defined by $\widetilde{y}=D^{\alpha} u=D\left[I^{1-\alpha} u\right]$, and a careful computation shows that the following inputoutput representation holds:

$$
\widetilde{y}(t)=\int_{0}^{\infty} \mu_{1-\alpha}(\xi)\left[u-\xi e_{\xi} \star u\right](t) \mathrm{d} \xi .
$$

The following infinite-dimensional dynamical system can be seen as a state-space realization of the fractional derivative of order $\alpha$ :

$$
\begin{aligned}
\partial_{t} \widetilde{\varphi}(\xi, t) & =-\xi \widetilde{\varphi}(\xi, t)+u(t), \widetilde{\varphi}(\xi, 0)=0 \\
\widetilde{y}(t) & =\int_{0}^{\infty} \mu_{1-\alpha}(\xi)[u(t)-\xi \widetilde{\varphi}(\xi, t)] \mathrm{d} \xi
\end{aligned}
$$

\section{Appendix B. FOURIER ANALYSIS OF THE LINEAR} MODEL

\section{B.1 Full model}

The Fourier transforms in time and space are denoted by $\widehat{w}(\omega)=\int_{-\infty}^{+\infty} w(t) e^{-i \omega t} \mathrm{~d} t, \quad \widehat{w}(k)=\int_{-\infty}^{+\infty} w(x) e^{+i k x} \mathrm{~d} x$ where $\omega$ is the angular frequency and $k$ is the wavenumber. Applying these transforms to the fractional equation (1) provides the nonlinear equation

$$
i \omega \hat{u}-i k\left(c \hat{u}+\frac{b}{2} \widehat{u^{2}}\right)+\chi \hat{u}=0
$$

with the fractional derivative coefficient

$$
\chi(\omega)=\varepsilon(i \omega)^{\alpha} .
$$

When $c \neq 0$ and $b=0$, one obtains the dispersion relation

$$
k=\frac{\omega}{c}-i \frac{\varepsilon}{c}(i \omega)^{\alpha} .
$$

It follows the phase velocity $v:=\omega / \Re \mathrm{e}(k)$ and the attenuation $\beta_{a}:=-\Im \mathrm{m}(k)$ :

$$
v(\omega)=\frac{c}{1+\varepsilon \sin \left(\frac{\alpha \pi}{2}\right) \omega^{\alpha-1}}, \quad \beta_{a}=\frac{\varepsilon}{c} \cos \left(\frac{\alpha \pi}{2}\right) \omega^{\alpha} .
$$

One deduces the elementary properties:

$$
\begin{aligned}
& v(0)=0, \quad \lim _{\omega \rightarrow+\infty} v(\omega)=c, \quad v^{\prime}>0, \\
& \beta_{a}(0)=0, \quad \lim _{\omega \rightarrow+\infty} \beta_{a}(\omega)=+\infty,
\end{aligned}
$$

that are shown on top of figure B.1.

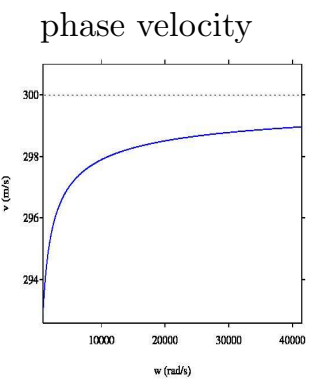

phase velocity (optim.)

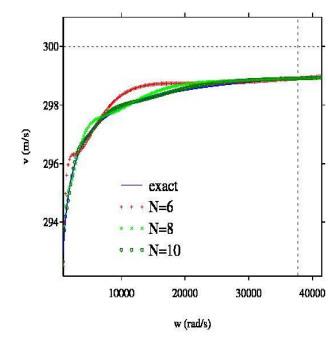

attenuation

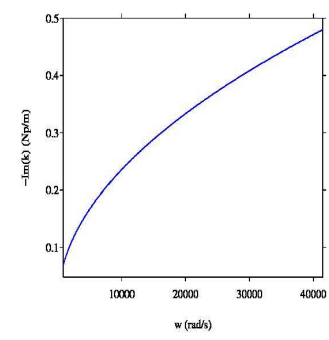

attenuation (optim.)

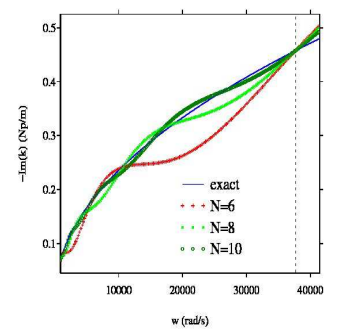

Fig. B.1. Dispersion curves in the linear regime $(b=0)$, with $c=300 \mathrm{~m} / \mathrm{s}, \varepsilon=1$ and $\alpha=1 / 2$. Comparison between the original model (top) with fractional derivatives (1) and the diffusive representation model (6) where the coefficients are determined by optimization (bottom). The horizontal dotted line denotes $c$. The vertical dotted lines denote the range of optimization $\left[\omega_{\min }, \omega_{\max }\right]$.

\section{B.2 Discretized model}

Still when $b=0$, a Fourier analysis can be performed on (6). A relation similar to (B.3) is obtained, changing $\chi$ by the diffusive representation coefficient

$$
\tilde{\chi}(\omega)=\varepsilon \gamma_{\alpha} \sum_{\ell=1}^{N} \mu_{\ell} \theta_{\ell}^{2 \alpha-1} \frac{i \omega}{\theta_{\ell}^{2}+i \omega}
$$

for which dispersion curves are shown on bottom of figure B.1. 